\title{
BRD4 promotes pancreatic ductal adenocarcinoma cell proliferation and enhances gemcitabine resistance
}

\author{
YONG-HUI WANG ${ }^{1}$, YA-NA SUI ${ }^{2}$, KAI YAN $^{1}$, LI-SHAN WANG $^{1}$, FEI WANG $^{3}$ and JIA-HUA ZHOU ${ }^{1}$ \\ ${ }^{1}$ Department of General Surgery, The Affiliated Zhongda Hospital, Medical School, Southeast University, Nanjing, Jiangsu; \\ ${ }^{2}$ Emergency Department of Weifang Traditional Chinese Hospital, Weifang, Shandong; ${ }^{3}$ Department of Hematology \\ and Oncology (Key Department of Jiangsu Medicine), Medical School, Zhongda Hospital, \\ Southeast University, Nanjing, Jiangsu, P.R. China
}

Received November 5, 2014; Accepted December 19, 2014

DOI: $10.3892 /$ or.2015.3774

\begin{abstract}
Pancreatic ductal adenocarcinoma (PDAC) is a highly aggressive carcinoma with a poor prognosis. To date, there is no effective treatment for this fatal disease. The manipulation of epigenetic proteins, such as BRD4, has recently emerged as an alternative therapeutic strategy. Our objective was to analyze the effect of BRD4 on the cell progression and chemoresistance of PDAC and the novel mechanisms involved. In the present study, we firstly revealed that the expression of BRD4 was significantly upregulated in PDAC cell lines, compared to that in human pancreatic duct epithelial cells. An in vitro assay showed that the suppression of BRD4 impaired PDAC cell viability and proliferation. Similarly, the tumor growth rate was also decreased in vivo after silencing of BRD4. Furthermore, we showed that the expression of BRD4 was increased after treatment with gemcitabine (GEM). Combination treatment of GEM and BRD4 silencing had a synergistic effect on the chemotherapeutic efficacy in the PANC-1 and MIAPaCa- 2 cell lines, and significantly promoted apoptosis. In particular, we demonstrated that BRD4 activated the Sonic hedgehog (Shh) signaling pathway members in a ligand-independent manner in the PDAC cells. Together, our results indicate the important role of BRD4 in PDAC cell proliferation and chemoresistance and suggests that BRD4 is a promising target directed against the transcriptional program of PDAC.
\end{abstract}

\section{Introduction}

Pancreatic ductal adenocarcinoma (PDAC) is the fourth leading cause of cancer-related death in the USA (1). Significant advances in the therapy for PDAC have been achieved in the

Correspondence to: Dr Jia-Hua Zhou, Department of General Surgery, The Affiliated Zhongda Hospital, Medical School, Southeast University, 87 Dingjiaqiao, Nanjing, Jiangsu, P.R. China

E-mail: zhoujh@seu.edu.cn

Key words: BRD4, chemoresistance, pancreatic cancer, proliferation, Sonic hedgehog pathway past few decades; however, the overall 5-year survival is still less than $5 \%$ (2). The dismal prognosis is particularly due to the occult early symptoms and the difficulty in early diagnosis. Thus, less than $20 \%$ of tumors are resectable at the time of diagnosis (3). Recurrence is common for patients who undergo surgical intervention. Chemotherapy is the most important adjuvant treatment for pancreatic cancer patients who are recurrent and not indicated for resection. However, PDAC is also notoriously resistant to gemcitabine (GEM), which is the first-line chemotherapeutic agent. Therefore, it is of utmost importance to identify new molecular targets for the effective treatment of pancreatic cancer.

Epigenetics is a rapidly progressing field of oncology. Modulation of epigenetic regulators has emerged as an alternative therapeutic approach in cancer treatment. BRD4 belongs to the bromodomain and extraterminal domain (BET) family that contains two bromodomains in tandem and an extra terminal domain. As a conserved epigenome regulator, BRD4 regulates the expression of numerous genes involved in the cell cycle, cell growth and inflammation $(4,5)$. Recent studies have demonstrated that BRD4 occupies super-enhancers of key oncogenic genes in certain biological contexts $(6,7)$. Oncogenic genes regulated by super-enhancers are greatly sensitive to levels of BRD4. Several studies have found that inhibition of BRD4 resulted in significant downregulation of c-Myc in hematopoietic malignancies and glioblastoma $(8,9)$. In lung adenocarcinoma, FOSL1 has been demonstrated as the main effector of BRD4 (10). Whether there is a different role for BRD4 in different cell lines is unclear. A recent study found that silencing of BRD4 in PDAC cells decreased the cell growth and abolished epithelial-to-mesenchymal transition (11). However, the widespread and heterogeneous genetic aberrations that occur in PDAC remain a major challenge, and cause a decrease in the response of PDAC to treatment targeting these highly altered signaling pathways. In addition, the mechanism by which BRD4 takes part in the regulation of PDAC cell proliferation remains elusive. Therefore, there is a vital necessity to illustrate the function and mechanism of BRD4 in regards to PDAC.

In the present study, we demonstrated that BRD4 is involved in PDAC tumorigenesis. Specifically, BRD4 knockdown suppressed tumor growth both in vitro and in vivo. Our 
findings revealed the role of BRD4 in PDAC drug resistance. The genetic inhibition of BRD4 was found to increase GEM sensitivity. Previous studies have demonstrated that the Sonic hedgehog (Shh) pathway induces PDAC tumorigenesis by promoting cell proliferation and increasing drug resistance (12-14). Therefore, we aimed to elucidate whether BRD4 is associated with the Shh pathway in PDAC and to explore the mechanisms by which BRD4 enhances the proliferation and viability of PDAC cells. The present study demonstrated that BRD4 promoted the Shh signaling pathway in PDAC cells in a ligand-independent manner.

\section{Materials and methods}

Cell culture. Human PDAC cell lines (PANC-1, SW1990, COLO357, L3.6PL, Capan-2 and MIAPaCa-2) from Shanghai Cell Bank (Shanghai, China) were grown in Dulbecco's modified Eagle's medium (DMEM) supplemented with $10 \%$ fetal bovine serum (FBS), $100 \mathrm{U} / \mathrm{ml}$ penicillin and $0.1 \mathrm{mg} / \mathrm{ml}$ streptomycin. Additional $2.5 \%$ horse serum was used for the MIAPaCa-2 cells. Human pancreatic duct epithelial (HPDE) cells were maintained in keratinocyte serum-free medium (Gibco, Grand Island, NY, USA) supplemented with epidermal growth factor and bovine pituitary extract. The cells were cultured in a humidified incubator at $37^{\circ} \mathrm{C}$ with $5 \% \mathrm{CO}_{2}$. In some experiments, recombinant human Shh N-terminal peptide (rhShh) (R\&D Systems, Minneapolis, MN, USA) was added to the culture medium.

Establishment of cell lines stably expressing BRD4 shRNA. shRNA plasmids for human BRD4 were designed against the BRD4 gene and constructed in Phblv-u6-puro vectors. A nontarget scrambled oligonucleotide served as the negative control. To generate stable BRD4 knockdown cells, PANC-1 and MIAPaCa-2 cells were grown in 6-well plates until they reached $50 \%$ confluency. The medium was replaced with $1 \mathrm{ml}$ of fresh culture medium supplemented with $100 \mu \mathrm{l}$ viral supernatant $\left(1 \times 10^{8} \mathrm{UT} / \mathrm{ml}\right)$ and $8 \mu \mathrm{g} / \mathrm{ml}$ Polybrene for $24 \mathrm{~h}$. The PANC-1 and MIAPaCa-2 cells were further cultured in medium containing puromycin at 3 and $2 \mu \mathrm{g} / \mathrm{ml}$, respectively. Individual puromycin-resistant colonies were isolated during drug screening. Knockdown efficiency was assessed by immunoblotting. The shRNA sequences used in the present study were as follows: shBRD4,5'-GatccGCCTGGAGATGACATAG TCTTATTCAAGAGATAAGACTATGTCATCTCCAGGTT TTTTc-3' and shcontrol, 5'-GatccTTCTCCGAACGTGTCAC GTAATTCAAGAGATTACGTGACACGTTCGGAGAATT TTTTg-3'.

Cell viability assay. Cell viability was measured using the Cell Counting Kit-8 (Beyotime, Haimen, Jiangsu, China). Briefly, cells (3,000/well) in $200 \mu \mathrm{l}$ medium were plated into 96 -well plates. After culturing for various times, $10 \mu \mathrm{l}$ of CCK-8 solution was added into each well at $37^{\circ} \mathrm{C}$. After $2 \mathrm{~h}$, the optical density values of each well were measured using a microplate reader at $450 \mathrm{~nm}$.

Colony formation assay. PANC-1 and MIAPaCa-2 cells stably transfected with shBRD4 or shcontrol were seeded at 200 cells/well in 6-well plates. After 10 days of culture, the colonies were stained with crystal violet, photographed and then scored.

Cell apoptosis analysis. Cell apoptosis was assessed by flow cytometry. For the cell apoptosis assay, PANC-1 and MIAPaCa-2 cells stably transfected with shBRD4 or shcontrol were incubated in the absence or presence of $2 \mu \mathrm{mol} / 1 \mathrm{GEM}$ for 24 or $48 \mathrm{~h}$. The percentage of apoptotic cells was analyzed by staining with fluorescein isothiocyanate-conjugated Annexin V and propidium iodide (KeyGen Biotech, Nanjing, Jiangsu, China), immediately followed by flow cytometry.

Real-time RT-PCR. Total RNA from the cells was isolated using TRIzol reagent (Invitrogen, Carlsbad, CA, USA) and then reverse-transcribed using a reverse transcription kit (Takara, Dalian, China) following the manufacturer's protocol. Amplification specificity was verified by melting curve analysis and agarose gel electrophoresis. The relative mRNA expression of the target genes was calculated as the inverse log of $\Delta \Delta \mathrm{CT}$ and normalized to the reference gene $\beta$-actin. The primers used for amplification were: BRD4 forward, 5'-CATG GACATGAGCACAATCA-3' and reverse, 5'-TCATGGTCAG GAGGGTTGTA-3'; SHH forward, 5'-CCCAATTACAACCC CGACATC-3' and reverse, 5'-TCACCCGCAGTTTCACTC CT-3'; PTCH1 forward, 5'-TGAGACTGACCACGGCCTG-3' and reverse, 5'-ACCCTCAGTTGGAGCTGCTTC-3'; GLI1 forward, 5'-AGGGCTGCAGTAAAGCCTTCA-3' and reverse, 5'-CTTGACATGTTTTCGCAGCG-3'; $\beta$-actin forward, 5'-GA TCATTGCTCCTCCTGAGC-3' and reverse, 5'-ACTCCTGCT TGCTGATCCAC-3'.

Western blotting. Equal amounts of the protein from lysates of the cultured PDAC cells were subjected to $10 \%$ SDS-PAGE and then transferred to PVDF membranes. After blocking in 5\% skim milk, the membranes were incubated overnight at $4^{\circ} \mathrm{C}$ with the primary antibodies against BRD4 (Abcam, Cambridge, MA, USA), SHH, PTCH1, GLI1 (all from Santa Cruz Biotechnology, Santa Cruz, CA, USA) or $\beta$-actin (Beyotime, Haimen, Jiangsu, China). After washing thrice with washing buffer [phosphate-buffered saline (PBS) containing $0.1 \%$ Tween-20] for $5 \mathrm{~min}$, the membranes were incubated for $1 \mathrm{~h}$ with the secondary antibodies at room temperature. Specific bands were visualized with enhanced chemiluminescence reagent (KeyGen Biotech) on an autoradiographic film.

Immunohistochemistry. Paraffin-embedded tissue slides (4- $\mu \mathrm{m}$ thick) were deparaffinized in xylene, rehydrated through graded alcohol solutions, blocked in methanol containing $3 \%$ hydrogen peroxide, and then incubated with 1:200 rabbit anti-human anti-Ki67 antibody (Santa Cruz Biotechnology) at $4{ }^{\circ} \mathrm{C}$ overnight. After rinsing with PBS solution, secondary antibodies and streptavidin peroxidase complex reagent were applied for $1 \mathrm{~h}$ at room temperature. Finally, the sections were incubated in a 3,3'-diaminobenzidine solution at room temperature for $10 \mathrm{~min}$ and then counterstained with hematoxylin.

Animal studies. All animal studies were approved by the Animal Care and Welfare Committee of Southeast University and were conducted in strict accordance with the guidelines of the National Animal Welfare Law of China. Five-week old 


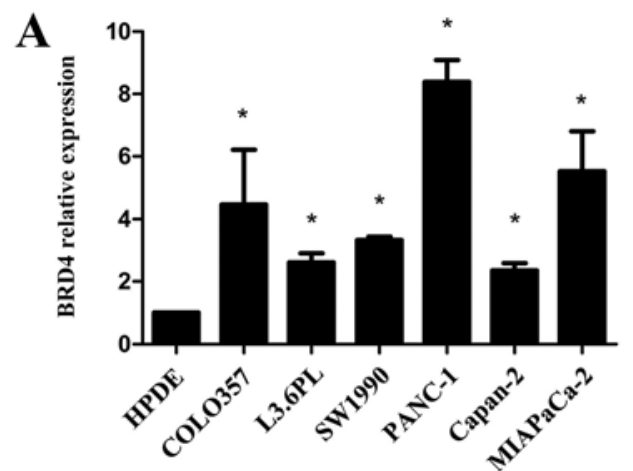

B

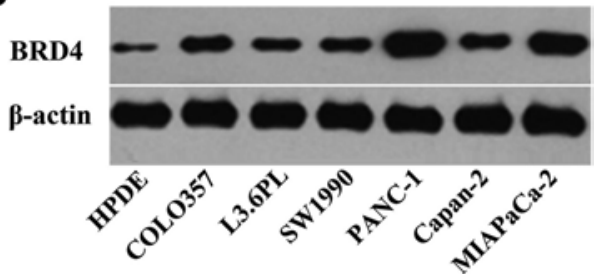

Figure 1. Identification of BRD4 upregulation in pancreatic cancer cell lines. (A) Expression of BRD4 mRNA levels in human pancreatic cancer cell lines, COLO357, L3.6PL, SW1990, PANC-1, Capan-2 and MIAPaCa-2, as assessed by real-time PCR. The human pancreatic duct epithelial (HPDE) cell line served as a control. (B) Overexpression of BRD4 in 6 pancreatic cancer cell lines compared to HPDE cells as assessed by western blotting.

A

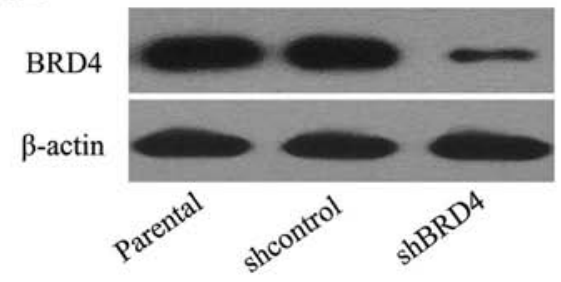

C

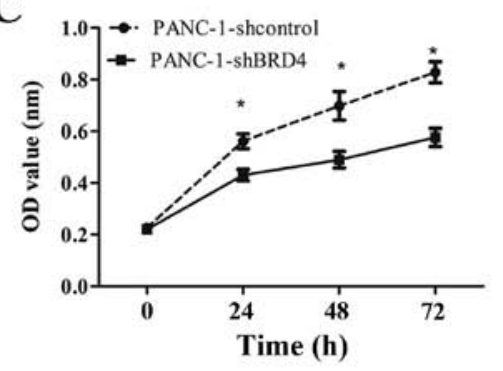

E
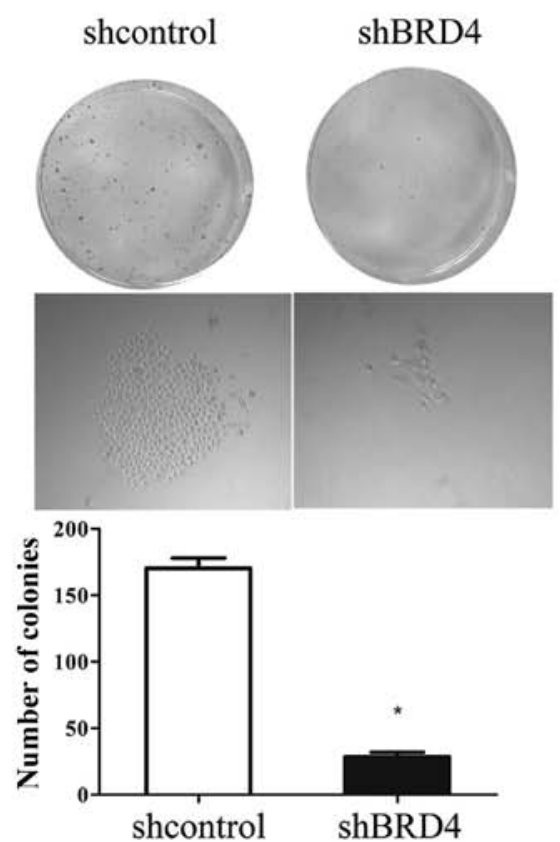

B

D

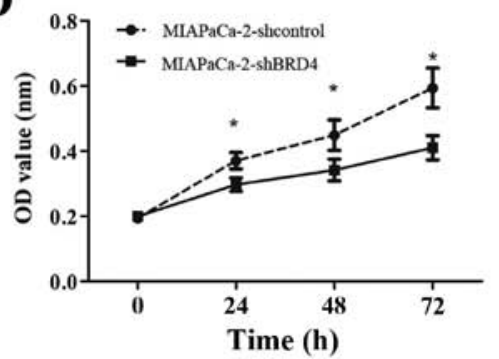

F

MIAPaCa-2
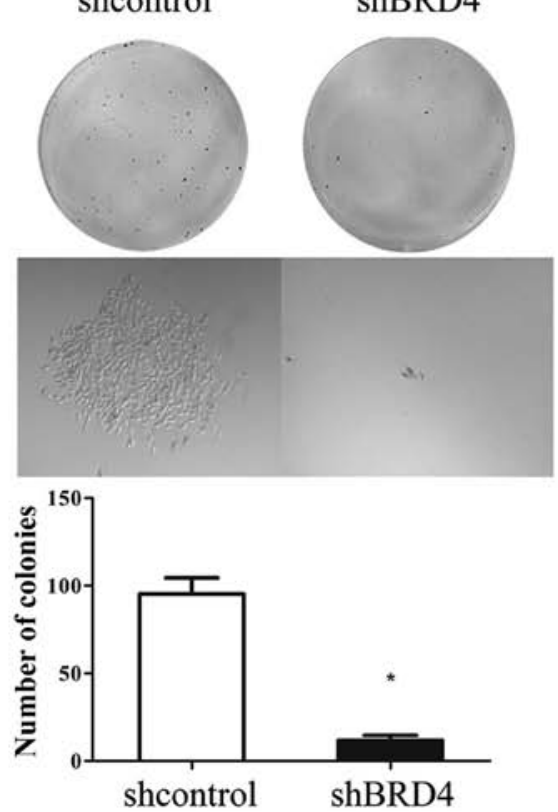

Figure 2. Knockdown of BRD4 inhibits the growth of pancreatic cancer cells. (A and B) Western blot analysis demonstrating the efficiency of BRD4 knockdown with shBRD4 in the (A) PANC-1 and (B) MIAPaCa-2 cell lines. (C and D) Effect of BRD4 shRNA induction on (C) PANC-1 and (D) MIAPaCa-2 cell viability over $72 \mathrm{~h}$ using the CCK-8 assay ( $\left.{ }^{*} \mathrm{p}<0.05\right)$. (E and F) Macroscopic and microscopic ( $\mathrm{x} 100$ magnification) images and quantification of colonies formed by the (E) PANC-1 and (F) MIAPaCa-2 cell lines transduced with shBRD4 or shcontrol ( $\left.{ }^{*} \mathrm{p}<0.05\right)$. 
A

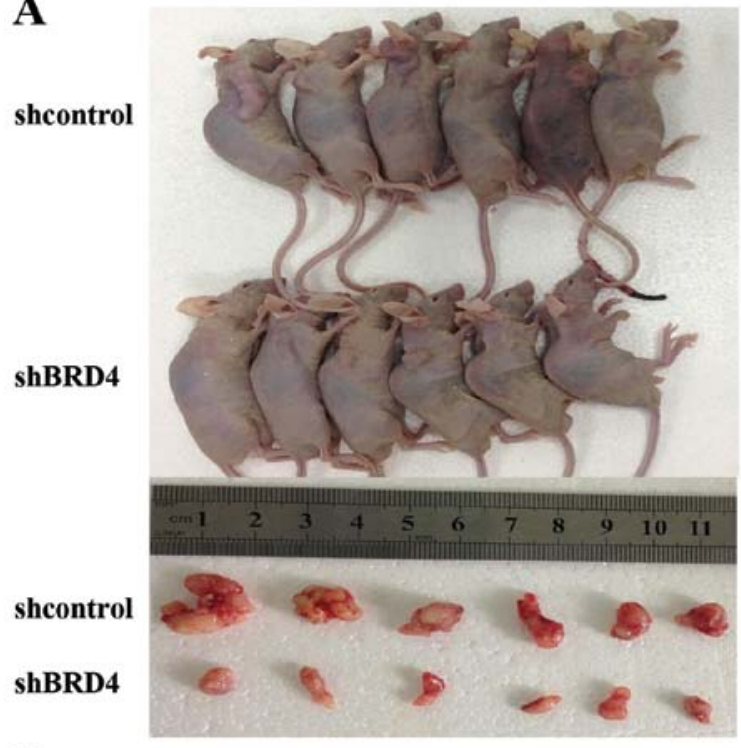

D

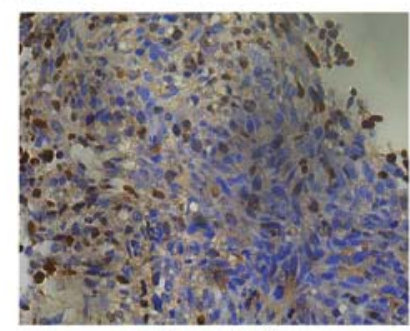

shcontrol

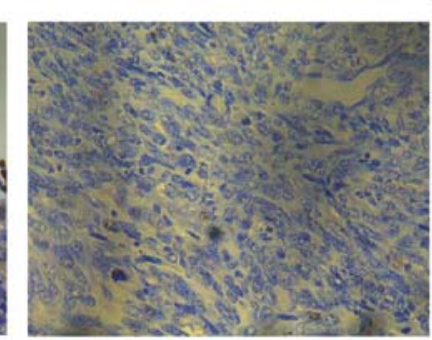

shBRD4
B

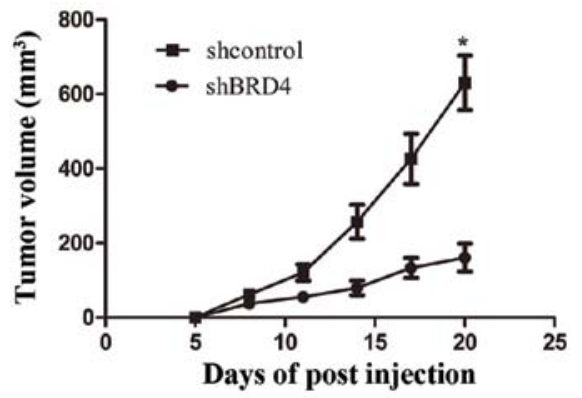

C

$\mathbf{E}$

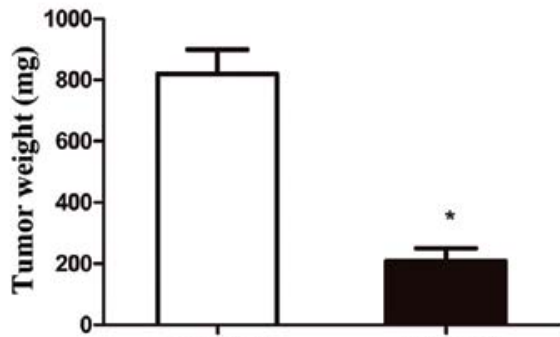

shcontrol

shBRD4

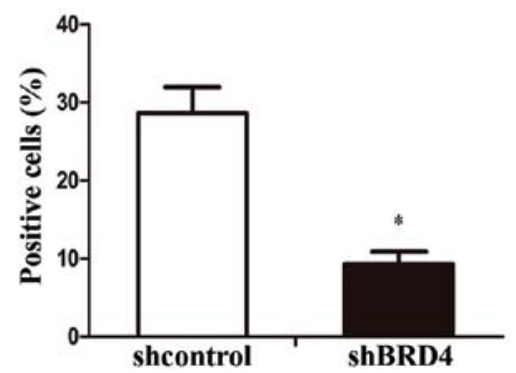

Figure 3. BRD4 maintains the tumorigenic capacity of PDAC in vivo. shcontrol- or shBRD4-transfected PANC-1 cells were injected subcutaneously into athymic nude mice ( $\mathrm{n}=6 /$ group). (A) Top panel: nude mice at 20 day postsubcutaneous implantation of the pancreatic tumor cells; bottom panel: macroscopic image of tumors excised from the mice in the top panel at the termination of the experiment. (B) The tumor volume of the shcontrol and shBRD4 tumors in mice was measured every 3 days ( $\left.{ }^{*} \mathrm{p}<0.05\right)$. (C) The average weight of the resected tumors from the bottom panel of A ( $\left.{ }^{*}<<0.05\right)$. (D) Representative images (x200 magnification) of pancreatic tumor sections of the shcontrol and shBRD4 PANC-1 tumors stained for Ki-67. (E) Quantification of the percentage of $\mathrm{Ki} 67(+)$ cells in the pancreatic tumor sections $\left({ }^{*} \mathrm{p}<0.05\right)$. PDAC, pancreatic ductal adenocarcinoma.

male BALB/c nude mice were procured from the Laboratory Animal Center of Yangzhou University (Yangzhou, China) and maintained in a specific pathogen-free environment. Stable PANC-1 shBRD4 and PANC-1 shcontrol cells $\left(2 \times 10^{6}\right)$ were subcutaneously injected into the right flanks of athymic nude mice. Tumor volume $\left(\mathrm{mm}^{3}\right)$ was calculated every 3 days for 3 weeks using the formula $\mathrm{V}=0.5 \mathrm{x}$ length $\mathrm{x}$ width ${ }^{2}$. After 3 weeks, the mice were euthanized. The tumors were isolated, weighed, photographed and then processed for immunohistochemistry.

Statistical analysis. All experiments were repeated in triplicate. Unless otherwise indicated, experimental values are expressed as mean \pm SEM. Statistical significance was determined by the unpaired Student's t-test using SPSS 13.0. $\mathrm{p}<0.05$ was considered to indicate a statistically significant result.

\section{Results}

$B R D 4$ is overexpressed in PDAC. To investigate the potential role of BRD4 in PDAC, we assessed the expression level of BRD4 in the PDAC cell lines COLO357, L3.6PL, SW1990, PANC-1, Capan-2 and MIAPaCa-2, as well as in the normal pancreatic epithelial cell line HPDE. qRT-PCR and western blot analyses showed that the mRNA and protein expression levels of BRD4 were significantly higher in the PDAC cells than these levels in the HPDE cells (Fig. 1A and B). The highest expression levels of BRD4 were detected in the PANC-1 and MIAPaCa-2 cells. The increased level of BRD4 in PDAC suggests that BRD4 promotes pancreatic tumorigenesis.

BRD4 promotes the viability and proliferation of pancreatic cancer cells in vitro. We downregulated BRD4 in the PANC-1 and MIAPaCa-2 cells to examine the influence of this protein on the biological behavior of PDAC cells. The expression level of BRD4 was confirmed by western blotting (Fig. 2A and B). PANC-1 and MIAPaCa-2 cells were selected for further studies due to their high expression levels of BRD4. The viability of the PANC-1 and MIAPaCa-2 cells was investigated using the CCK- 8 assay after 24,48 and $72 \mathrm{~h}$ of incubation. BRD4 knockdown evidently inhibited the viability of both cell lines (Fig. 2C and D). Furthermore, the colony formation assay showed that the proliferation of stable BRD4-knockdown cells was severely inhibited (Fig. 2E and F). These results suggest that BRD4 is involved in the growth of pancreatic cancer cells.

BRD4 is essential for pancreatic cancer progression in vivo. To study the relevance of BRD4 to PDAC progression in vivo, we 
A

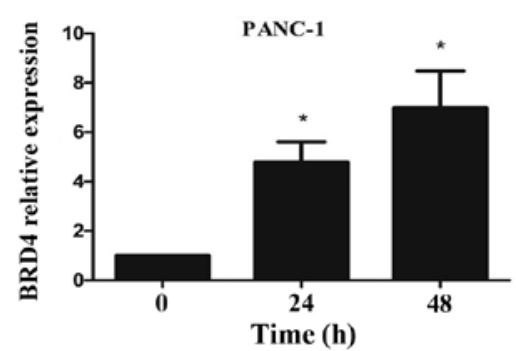

C

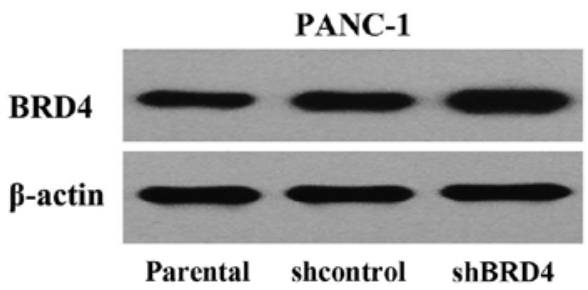

B

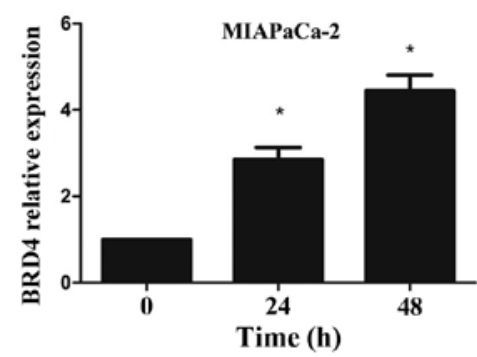

D

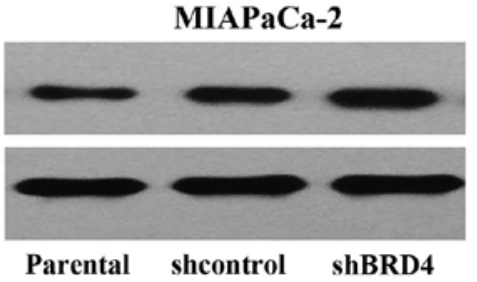

E
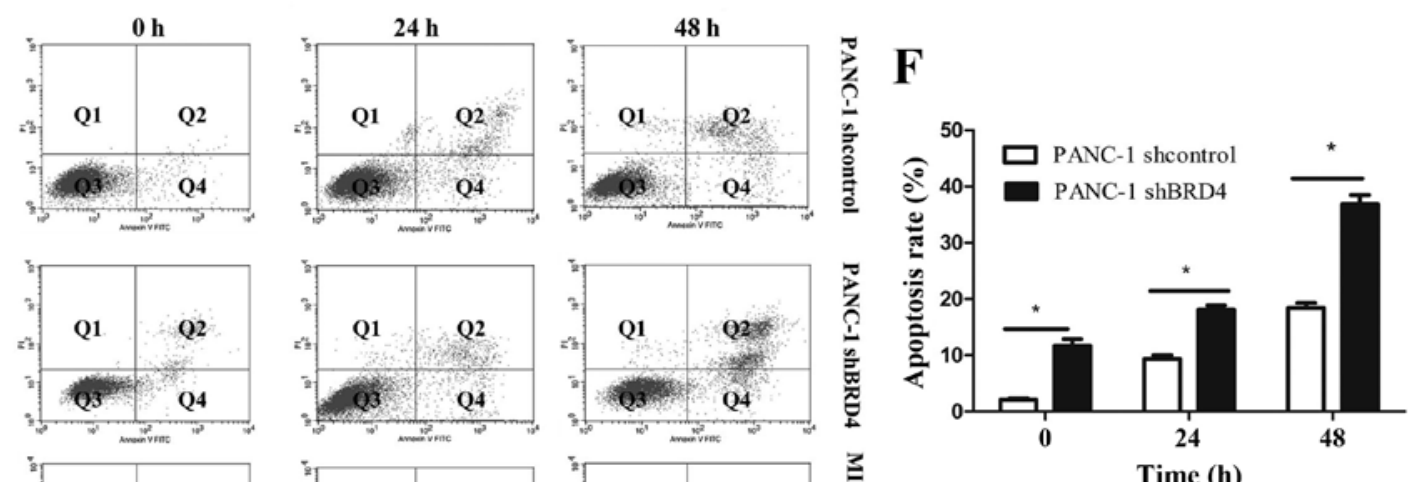

$\overline{2}$
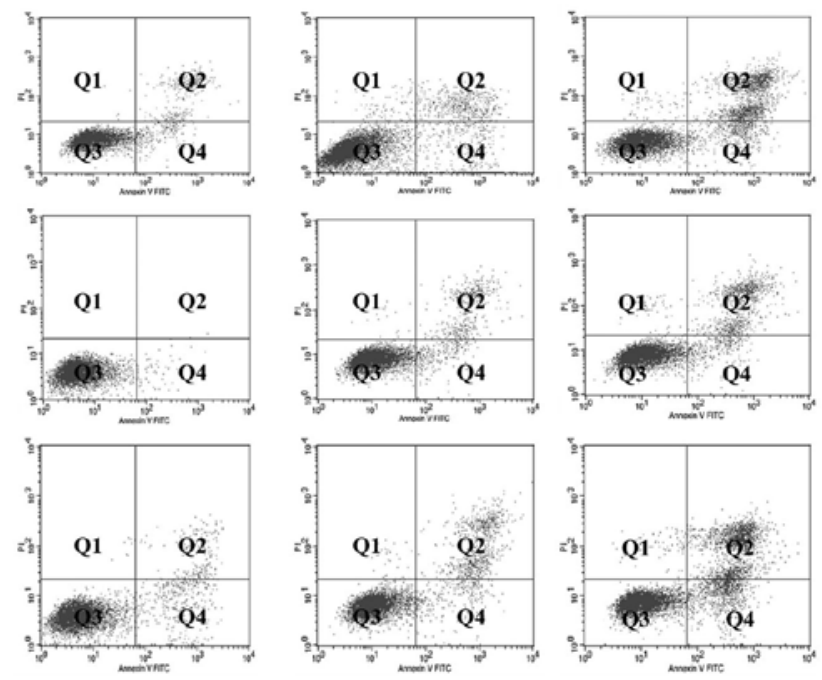

Annexin VFITC

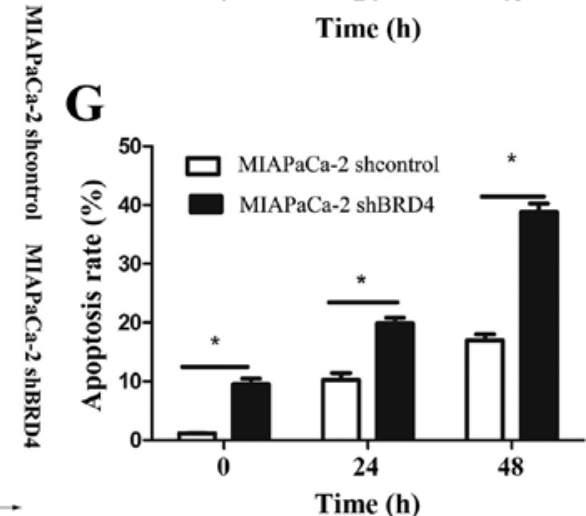

Figure 4. Inhibition of BRD4 by shRNA sensitizes PDAC cells to gemcitabine (GEM). (A-D) Induction of BRD4 in pancreatic cancer cell lines treated with GEM. PANC-1 and MIAPaCa-2 cells were incubated with $2 \mu \mathrm{mol} / 1$ GEM for 24 or $48 \mathrm{~h}$. (A and B) BRD4 expression was monitored by quantitative RT-PCR and (C and D) western blot analysis ("p<0.05). (E) After treatment with $2 \mu \mathrm{mol} / 1 \mathrm{GEM}$ for $24 \mathrm{or} 48 \mathrm{~h}$, the apoptosis rate was analyzed using flow cytometry. $\mathrm{Q}_{2}+\mathrm{Q}_{4}$ is indicative of apoptosis. ( $\mathrm{F}$ and $\left.\mathrm{G}\right)$ Quantification of the data from $\mathrm{E}\left({ }^{*} \mathrm{p}<0.05\right)$. PDAC, pancreatic ductal adenocarcinoma.

subcutaneously transplanted shBRD4 and shcontrol PANC-1 cells into nude mice (n=6/group). At 20 days after cell implantation, the tumors were removed and photographed (Fig. 3A). Consistent with a previous study, the present study showed that BRD4 silencing did not affect tumor initiation (15). However, the periodic measurements of tumor volume and the final weight of the excised tumors demonstrated that BRD4 shRNA-PANC-1 cells had significantly retarded tumor growth at termination compared with the control cells (Fig. 3B and C). Histologic analysis of tumor proliferation revealed that shBRD4 tumors had significantly fewer Ki-67-positive cells than the shcontrol tumors (Fig. 3D and E). These results indi- cate that BRD4 participates in PDAC proliferation and tumor maintenance in vivo.

BRD4 knockdown causes GEM sensitization. PANC-1 and MIAPaCa- 2 cells were treated with $2 \mu \mathrm{mol} / 1$ GEM to investigate the role of BRD4 in drug resistance. GEM significantly increased the mRNA and protein expression levels of BRD4 in the PANC-1 and MIAPaCa-2 cells (Fig. 4A-D). Then, we examined the cell apoptosis rate of PANC-1 and MIAPaCa-2 cells after treatment with $2 \mu \mathrm{mol} / \mathrm{l}$ GEM for 24 or $48 \mathrm{~h}$ to further elucidate the relationship between BRD4 and drug resistance in pancreatic cancer cells. BRD4 knockdown alone 
A

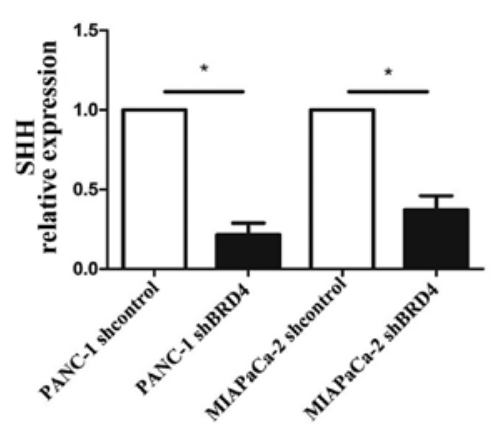

C

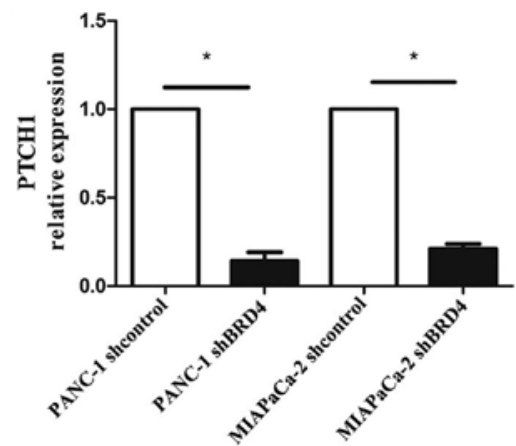

$\mathbf{E}$

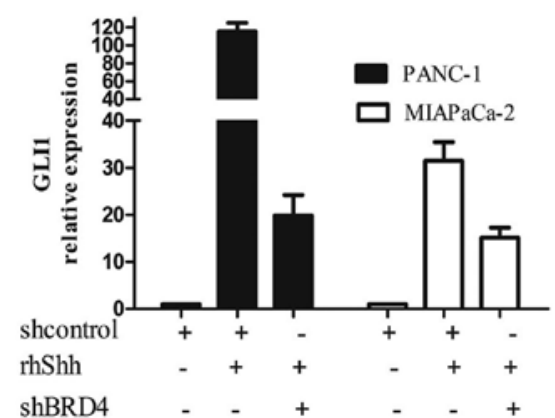

G

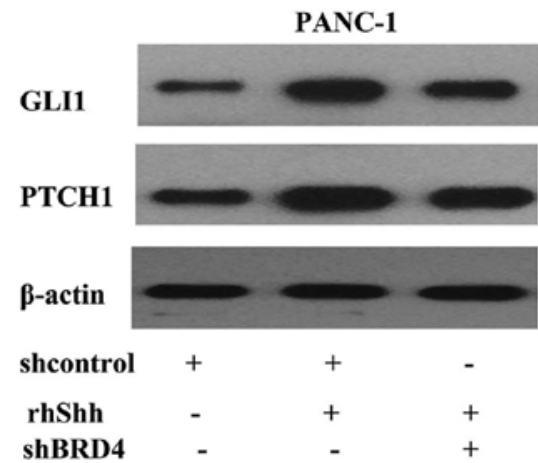

B
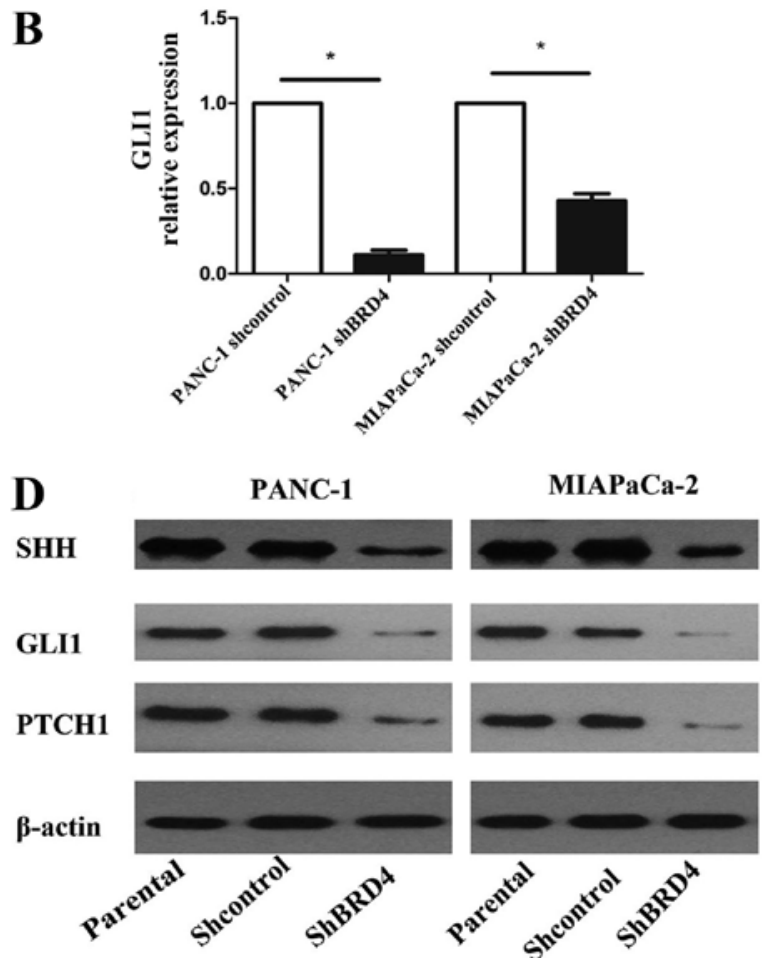

F
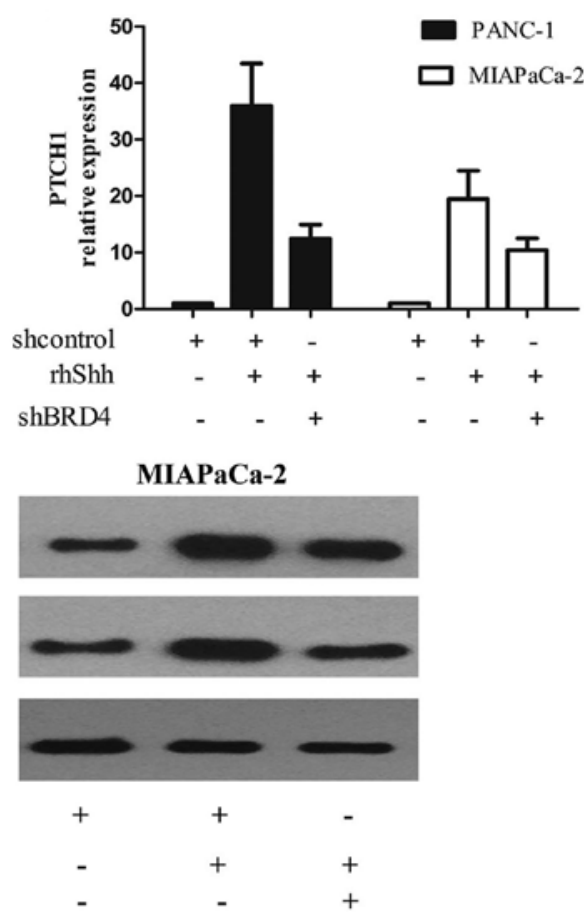

Figure 5. BRD4 upregulates the Shh signaling pathway. (A-C) qRT-PCR analysis of BRD4 in the transcriptional regulation of (A) Shh, (B) PTCH1 and (C) GLI1. mRNA levels were determined in the PANC-1-shBRD4 and MIAPaCa-2-shBRD4 cell lines and their control group cells. The relative levels were normalized to $\beta$-actin ( ${ }^{*} \mathrm{p}<0.05$ ). (D) Parallel samples from A-C were immunoblotted for the proteins of Shh, PTCH1 and GLI1. $\beta$-actin served as a loading control ( $" \mathrm{p}<0.05$ ). (E and F) After the PDAC cells were cultured with or without $0.5 \mu \mathrm{g} / \mathrm{ml}$ of rhShh for $24 \mathrm{~h}$, qRT-PCR analysis of Shh target genes (E) PTCH1 and (F) Gli1 was carried out. (G) Parallel samples from E and F were immunoblotted for the proteins of PTCH1 and GLI1. $\beta$-actin served as a loading control $($ ( $\mathrm{p}<0.05)$. PDAC, pancreatic ductal adenocarcinoma.

induced cell apoptosis in the PANC-1 and MIAPaCa-2 cells; however, treatment with GEM significantly promoted cell apoptosis (Fig. 4E-G). These results revealed that BRD4 decreased the GEM sensitivity of pancreatic cancer cells. In addition, BRD4 downregulation and GEM treatment synergistically influenced the chemotherapeutic efficacy in the PDAC cells.
$B R D 4$ regulates the Shh signaling pathway in pancreatic cancer cells. Recent study has shown that the Shh signaling pathway is regulated by BRD4 (16). The Shh signaling pathway plays a critical role in the genesis of PDAC, and maintenance of hedgehog signaling is important for aberrant proliferation and tumorigenesis $(12,13)$. We analyzed the 
expression of the principal components of the Shh signaling pathway in BRD4-knockdown PANC-1 and MIAPaCa-2 cells to further explore the mechanism by which BRD4 influences the proliferation and viability of pancreatic cancer cells. BRD4 knockdown decreased the mRNA and protein levels of Shh pathway-related genes, including SHH, PTCH1 and GLI1, in both cell lines (Fig. 5A-D). These results indicate that BRD4 promotes the Shh signaling pathway in pancreatic cancer cells in a ligand-dependent manner. Several in vitro experiments using cell lines indicated that the Shh signaling pathway is partially activated in PDAC in a ligand-independent manner $(17,18)$. Therefore, we investigated whether or not BRD4-induced Shh contributes to Shh signaling pathway activation. BRD4 knockdown in PANC-1 and MIAPaCa-2 cells followed by rhShh stimulation markedly inhibited Shh target gene expression (Fig. 5E-G). This result supports the essential role of BRD4 in the Shh signaling pathway. These data suggest that the BRD4-induced increase in Shh transcription does not contribute to Shh pathway activation. In other words, BRD4-induced Shh pathway activation is essentially independent of Shh.

\section{Discussion}

Epigenetic modifications in cancer initiation and progression have attracted increased attention. Targeting epigenetic modulators known as 'readers' is a promising cancer treatment strategy. As an epigenome reader, the BRD4 protein plays a critical role at the interface between chromatin remodeling and transcriptional regulation. Previous studies have described the therapeutic potential of targeting BRD4 in models of acute myeloid leukemia, glioblastoma, multiple myeloma, lung adenocarcinoma and osteosarcoma $(8-10,19,20)$. By contrast, BRD4 is downregulated in breast and colon cancers; thus, this protein may serve as a tumor suppressor in these cancers $(21,22)$. Whether or not BRD4 is a tumor promoter or suppressor remains controversial. These contradictory results reveal that the function of BRD4 in different tissues depends on the type of cancer, the mechanism of tumorigenesis, and the abundance of different BRD4 downstream targets. Therefore, future research must demonstrate the conclusive function of BDR4 in different types of cancers. In the present study, BRD4 was overexpressed in PDAC cells. Consistent with a recent study (11), we also showed that BRD4 promoted the viability and proliferation of pancreatic cancer cells in vitro and this was further illustrated in vivo. These results suggest that BRD4 is crucial in pancreatic cancer tumorigenesis.

PDAC is a fatal disease due to its aggressiveness and resistance to chemotherapy. Selective inhibition targeting small-molecule proteins, together with effective conventional chemotherapeutic agents, is a promising approach to combat cancer chemoresistance. In the present study, GEM significantly upregulated BRD4 expression in PDAC cell lines. This upregulation may be involved in the adaptive response to DNA damage in PDAC chemotherapy. We also found that BRD4 knockdown in PDAC cells enhanced the apoptotic effect of GEM, further verifying our hypothesis regarding the involvement of BRD4 in chemoresistance. These data suggest that BRD4 inhibition and GEM treatment synergistically influence pancreatic cancer treatment.
The epigenetic state of a cell is markably influenced by the cell line. Thus, chromatin-binding proteins possibly have different transcriptional targets in diverse types of cancer. BET family members induce specific subsets of gene expression in different cell types. For example, the BET protein in cell lineage derived from hematologic malignancies promotes cell cycle progression by affecting MYC gene expression (23). In acute lymphoblastic leukemia, this protein induces the same effect by influencing c-Myc and IL7R gene expression (8). However, a recent study has reported that cyclin D1 is the principal effector of BET protein-induced cell-cycle progression in malignant peripheral nerve sheath tumors (19). In lung adenocarcinoma, instead of MYC or cyclin D1, FOSL1 plays the same role in cell cycle progression (10). BRD4 silencing represses not only FOSL1 and c-Myc expression in PDAC yet also HMGA2, an architectural protein that we previously identified to mediate chemoresistance in pancreatic cancer cells (11). The present study demonstrated that BRD4 induces PDAC cell viability and proliferation by regulating the Shh signaling pathway, which enhances PDAC cell proliferation and GEM resistance (12-14). Mammals have three known paralogous genes, namely, Shh, Indian hedgehog and Desert hedgehog (24). Among these genes, Shh is specifically overexpressed in PDAC (14). The Shh signaling pathway is initiated by the binding of the secreted Shh ligands to PTCH1 that relieves the PTCH1-mediated repression of SMO. SMO triggers the downstream signaling cascades that induce the nuclear translocation of Gli and consequently activates the transcription of Shh target genes, including PTCH1, Gli1, cyclin D and E and MYC. Aberrant Shh signaling activity is transduced through three signal transduction models: i) ligand-independent signaling; ii) ligand-dependent autocrine/juxtacrine signaling; and iii) ligand-dependent paracrine signaling (25). In the present study, BRD4 activated the Shh signaling pathway of PDAC in a ligand-independent manner. This finding suggests that BRD4 promotes the malignant transformation and drug resistance of PDAC cells by activating the Shh signaling pathway.

In conclusion, BRD4 promotes the proliferation and GEM resistance of PDAC possibly by regulating the Shh signaling pathway. Therefore, BRD4 is a novel target for the development of alternative therapeutic approaches for PDAC.

\section{Acknowledgements}

This study was financially supported by China's National Natural Science Foundation (81071967 and 30872500).

\section{References}

1. Siegel R, Ma J, Zou Z and Jemal A: Cancer statistics, 2014. CA Cancer J Clin 64: 9-29, 2014.

2. Li D, Xie K, Wolff R and Abbruzzese JL: Pancreatic cancer. Lancet 363: 1049-1057, 2004.

3. Davis JL, Pandalai PK, Ripley RT, Langan RC and Avital I: Expanding surgical treatment of pancreatic cancer: the role of regional chemotherapy. Pancreas 41: 678-684, 2012.

4. Yang Z, He N and Zhou Q: Brd4 recruits P-TEFb to chromosomes at late mitosis to promote $\mathrm{G}_{1}$ gene expression and cell cycle progression. Mol Cell Biol 28: 967-976, 2008.

5. Nicodeme E, Jeffrey KL, Schaefer U, et al: Suppression of inflammation by a synthetic histone mimic. Nature 468: 1119-1123, 2010. 
6. Loven J, Hoke HA, Lin CY, et al: Selective inhibition of tumor oncogenes by disruption of super-enhancers. Cell 153: 320-334, 2013.

7. Chapuy B, McKeown MR, Lin CY, et al: Discovery and characterization of super-enhancer-associated dependencies in diffuse large B cell lymphoma. Cancer Cell 24: 777-790, 2013.

8. Ott CJ, Kopp N, Bird L, et al: BET bromodomain inhibition targets both c-Myc and IL7R in high-risk acute lymphoblastic leukemia. Blood 120: 2843-2852, 2012.

9. Pastori C, Daniel M, Penas C, et al: BET bromodomain proteins are required for glioblastoma cell proliferation. Epigenetics 9: 611-620, 2014.

10. Lockwood WW, Zejnullahu K, Bradner JE and Varmus H: Sensitivity of human lung adenocarcinoma cell lines to targeted inhibition of BET epigenetic signaling proteins. Proc Natl Acad Sci USA 109: 19408-19413, 2012.

11. Sahai V, Kumar K, Knab LM, et al: BET bromodomain inhibitors block growth of pancreatic cancer cells in three-dimensional collagen. Mol Cancer Ther 13: 1907-1917, 2014.

12. Berman DM, Karhadkar SS, Maitra A, et al: Widespread requirement for Hedgehog ligand stimulation in growth of digestive tract tumours. Nature 425: 846-851, 2003.

13. Thayer SP, di Magliano MP, Heiser PW, et al: Hedgehog is an early and late mediator of pancreatic cancer tumorigenesis. Nature 425: 851-856, 2003.

14. Xu M, Li L, Liu Z, et al: ABCB2 (TAP1) as the downstream target of SHH signaling enhances pancreatic ductal adenocarcinoma drug resistance. Cancer Lett 333: 152-158, 2013.

15. Segura MF, Fontanals-Cirera B, Gaziel-Sovran A, et al: BRD4 sustains melanoma proliferation and represents a new target for epigenetic therapy. Cancer Res 73: 6264-6276, 2013.

16. Tang Y, Gholamin S, Schubert S, et al: Epigenetic targeting of Hedgehog pathway transcriptional output through BET bromodomain inhibition. Nat Med 20: 732-740, 2014.
17. Onishi $\mathrm{H}$, Kai M, Odate $\mathrm{S}$, et al: Hypoxia activates the hedgehog signaling pathway in a ligand-independent manner by upregulation of Smo transcription in pancreatic cancer. Cancer Sci 102: 1144-1150, 2011.

18. Nolan-Stevaux O, Lau J, Truitt ML, et al: GLII is regulated through Smoothened-independent mechanisms in neoplastic pancreatic ducts and mediates PDAC cell survival and transformation. Genes Dev 23: 24-36, 2009.

19. Patel AJ, Liao CP, Chen Z, Liu C, Wang Y and Le LQ: BET bromodomain inhibition triggers apoptosis of NF1-associated malignant peripheral nerve sheath tumors through Bim induction. Cell Rep 6: 81-92, 2014.

20. Lamoureux F, Baud'huin M, Rodriguez Calleja L, et al: Selective inhibition of BET bromodomain epigenetic signalling interferes with the bone-associated tumour vicious cycle. Nat Commun 5: $3511,2014$.

21. Rodriguez RM, Huidobro C, Urdinguio RG, et al: Aberrant epigenetic regulation of bromodomain BRD4 in human colon cancer. J Mol Med 90: 587-595, 2012.

22. Crawford NP, Alsarraj J, Lukes L, et al: Bromodomain 4 activation predicts breast cancer survival. Proc Natl Acad Sci USA 105: 6380-6385, 2008.

23. Mertz JA, Conery AR, Bryant BM, et al: Targeting MYC dependence in cancer by inhibiting BET bromodomains. Proc Natl Acad Sci USA 108: 16669-16674, 2011.

24. Geissler K and Zach O: Pathways involved in Drosophila and human cancer development: the Notch, Hedgehog, Wingless, Runt, and Trithorax pathway. Ann Hematol 91: 645-669, 2012.

25. Teglund S and Toftgård R: Hedgehog beyond medulloblastoma and basal cell carcinoma. Biochim Biophys Acta 1805: 181-208, 2010. 